\title{
Characterization of the compact Hokuyo URG-04LX 2D laser range scanner
}

\author{
Laurent Kneip, Fabien Tâche, Gilles Caprari, and Roland Siegwart \\ Autonomous Systems Laboratory, ETH Zurich
}

\begin{abstract}
This paper presents a detailed characterization of the Hokuyo URG-04LX 2D laser range finder. While the sensor specifications only provide a rough estimation of the sensor accuracy, the present work analyzes issues such as time drift effects and dependencies on distance, target properties (color, brightness and material) as well as incidence angle. Since the sensor is intended to be used for measurements of a tubelike environment on an inspection robot, the characterization is extended by investigating the influence of the sensor orientation and dependency on lighting conditions. The sensor characteristics are compared to those of the Sick LMS 200 which is commonly used in robotic applications when size and weight are not critical constraints. The results show that the sensor accuracy is strongly depending on the target properties (color, brightness, material) and that it is consequently difficult to establish a calibration model. The paper also identifies cases for which the sensor returns faulty measurements, mainly when the surface has low reflectivity (dark surfaces, foam) or for high incidence angles on shiny surfaces. On the other hand, the repeatability of the sensor seems to be competitive with the LMS 200.
\end{abstract}

\section{INTRODUCTION}

On the background of the development of a tube inspection robot [1] with a corresponding sensing system that serves for mapping and localization, this paper contributes to the characterization of a compact $2 \mathrm{D}$ laser range scanner, the Hokuyo URG-04LX [2]. The different experiments that have been realized to characterize the range finder have largely been inspired by $\mathrm{Ye}$ and Borenstein [3]. They propose a characterization with subsequent calibration model of a competitive, but larger and heavier planar range finder, the Sick LMS 200. Although there are already some works that try to benefit from the small size and the low weight of the URG-04LX [4], a proper characterization of the sensor that exploits the true potential and the limitations following Borenstein's method is still missing. For completeness, note

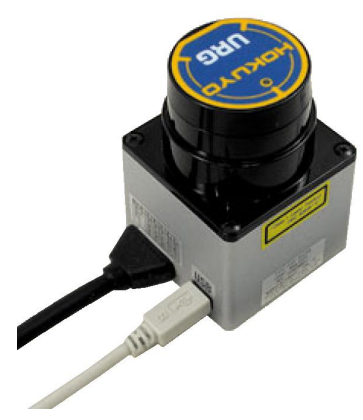

Fig. 1. The Hokuyo URG-04LX. that a comparison of both sensors was done by Lee [10]. However it does not drive useful and detailed conclusions for the use of the sensor and mainly addresses time drift effects. The present paper then provides a detailed and complete characterization of the sensor by checking the commonly known issues with 2D laser range finders [5] and their dependencies on, for example, target surface properties, distance, and incidence angle.

The idea behind the use of the Hokuyo sensor is obvious: 2D LADARs are popular sensing systems for challenging mapping, localization or obstacle negotiation tasks. Moving the sensor extends it to a 3D-range measurement system able to capture the "surrounding world" [6], [7]. It represents a reliable solution with a larger FOV than other 3D sensors like for example the solid-state flash LADAR Swiss Ranger [8] or stereo vision concepts.

The Hokuyo URG-04LX, shown in Figure 1, certainly fills a gap by providing a range measurement system for compact mobile robot applications. Very often, these need highly specialized devices with critical requirements regarding size and weight. The inspection robot for that the present work has been developed, is for example able to drive across tube structures having a diameter down to only $200 \mathrm{~mm}$ [1]. Moreover, the robot movement may lead to any orientation of the sensor system with respect to the path sections. Therefore, the usual experiments known from the works of Ye and Borenstein [3] and Alwan [9] have been extended with some specialized tests to check the dependency of the measurement on the orientation of the sensor or the influence of the ambient light.

The final goal of our investigation is to find out how far the Hokuyo URG-04LX can serve for building up a compact 3D-LADAR system without too much performance loss in comparison to the commonly used Sick LMS 200. Sections II and III start with presenting some sensor specifications and the experimental setup used to make the measurements. Section IV then presents the results of the experiments. The paper investigates drift effects and influence of sensor orientation, ambient light, surface color, surface brightness, material properties, distance and incidence angle. Finally the possibility of a calibration model for the Hokuyo sensor is analyzed in section V.

\section{THE HOKUYo URG-04LX LASER SCANNER}

The Hokuyo URG-04LX 2D laser scanner works different from the Sick sensor that is based on the ToF (Time of Flight) measurement principle [2]. It uses amplitude modulated 


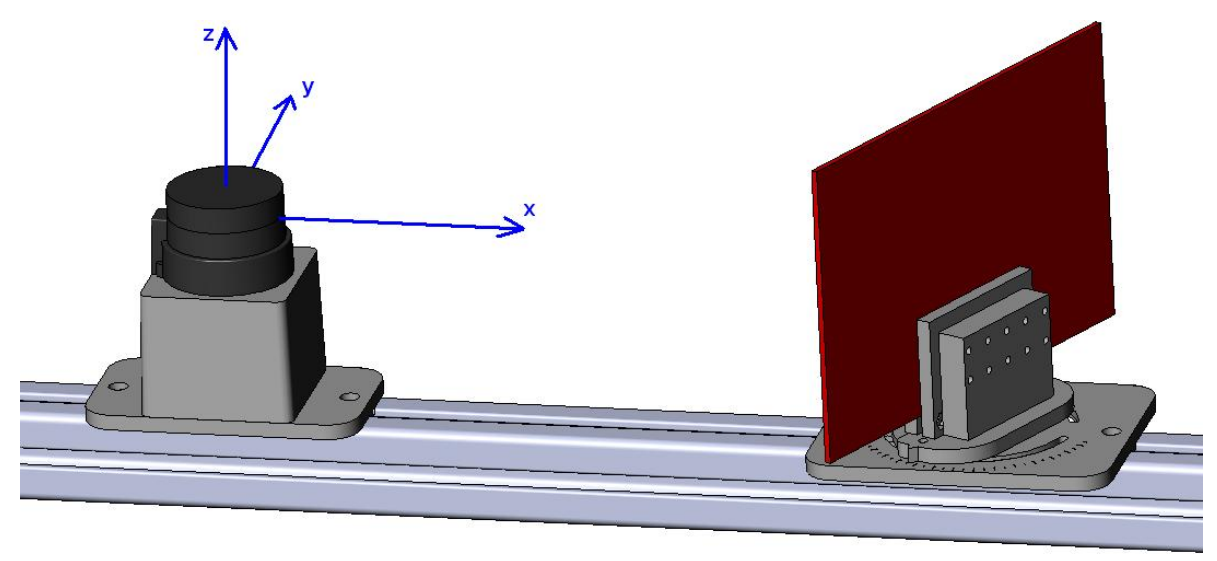

Fig. 2. CAD design of the supporting parts for the URG-04LX and the target surface.

laser light and deduces the target distance from the phase shift measurement between the emitted light wave and its reflection. It is a very compact and innovative laser range finder that differs from the LMS 200 mainly because of weight and size. It has been developed a few years ago by the Intelligent Robot Laboratory of the University of Tsukuba and the department of engineering of the Hokuyo Automatic Co., Ltd. in Japan. The inner life of the range scanner [2] consists of a compact stacked structure of a few control circuits with a spindle motor and the actual scanner on top of it. The motor is rotating a small transmission mirror that is deflecting the vertical class 1 laser beam coming from the top of the sensor into horizontal direction. This allows the laser beam to scan a planar area around the sensor with a broad opening angle of $240^{\circ}$. A second mirror below, the reception mirror, is again deviating the horizontal laser beam captured by a lens into vertical direction toward an Avalanche Photodiode (APD) sitting on top of the motor.

The compactness of the sensor is due to the usage of a brushless spindle motor with outer rotor. The size of the sensor equals to $50 \times 50 \times 70 \mathrm{~mm}$ and it weighs only $160 \mathrm{~g}$. The modulation frequencies allow the sensor to measure distances between $2 \mathrm{~cm}$ and $5.6 \mathrm{~m}$. The sensor alternately uses two different modulations which help to overcome ambiguities inherent to phase-modulated range measurements. The detector measures 683 steps on $240^{\circ}$ and has therefore a resolution of about $0.36^{\circ}$. The spindle motor is only rotating at $600 \mathrm{rpm}$, thus complete scans are taken with a frequency of $10 \mathrm{~Hz}$. The URG-04LX disposes over two interfaces for data transfer to the host: RS232 with data transfer rates up to $700 \mathrm{kbps}$ and full-speed USB.

Following the specification sheet of the sensor [11], the measurement accuracy should be $\pm 10 \mathrm{~mm}$ between $20 \mathrm{~mm}$ and $1 \mathrm{~m}$ and $\pm 1 \%$ of the measurement value for distances between 1 and $4 \mathrm{~m}$ for a white sheet of paper.

\section{EXPERIMENTAL SETUP}

Figure 2 shows the experimental setup that has been used for effectuating the characterization of the sensor, a rather simple configuration allowing manual setting of the desired surface, distance and incidence angle. It consists of a level track with fixed custom supporting parts for the Hokuyo URG-04LX as well as a clamp for a plate that carries the target surfaces. The track consists of a standardized aluminum profile. Adequate distance marks offer an easy way to slide the target along the track and set up the desired distance to the Hokuyo sensor with an accuracy of $1 \mathrm{~mm}$. The fixation for the surface can be rotated about a vertical axis and a scale allows an easy configuration of desired incidence angles between $-75^{\circ}$ and $75^{\circ}$. This scale is included in the design and thus allows the configuration of the incidence angle with satisfying precision $\left( \pm 1^{\circ}\right)$.

The measurements have been performed at slightly elevated room temperature of around $25^{\circ} \mathrm{C}$ and during the day with additional artificial light emitted by halogen bulbs. The sensor was powered by a voltage generator that delivered exactly 5V. Measurement data has been sent over RS232 to the PC at a baud rate of $57.6 \mathrm{kbps}$, which is sufficient since the sensor is configured to measure only between angle steps 360 and 420 . Reception and subsequent processing of the data was done in a special LabVIEW virtual instrument that stores all the data together with a histogram of the measurement values (shown in this paper), the properties of the current configuration and computed values for mean and standard deviation. The standard number of measurements for each experiment equals to 5000. The histograms of the measurement values of each experiment frequently consist of only one lobe which is almost Gaussian. The mean value is calculated using

$$
\bar{x}=\frac{1}{N} \sum_{i=1}^{N} x_{i}
$$

and the standard deviation with

$$
\sigma=\sqrt{\frac{1}{N} \sum_{i=1}^{N}\left(x_{i}-\bar{x}\right)^{2}} .
$$

The relative error $\eta$ are computed using the formula

$$
\eta=\frac{d_{\text {measured }}-d_{\text {nominal }}}{d_{\text {nominal }}} .
$$




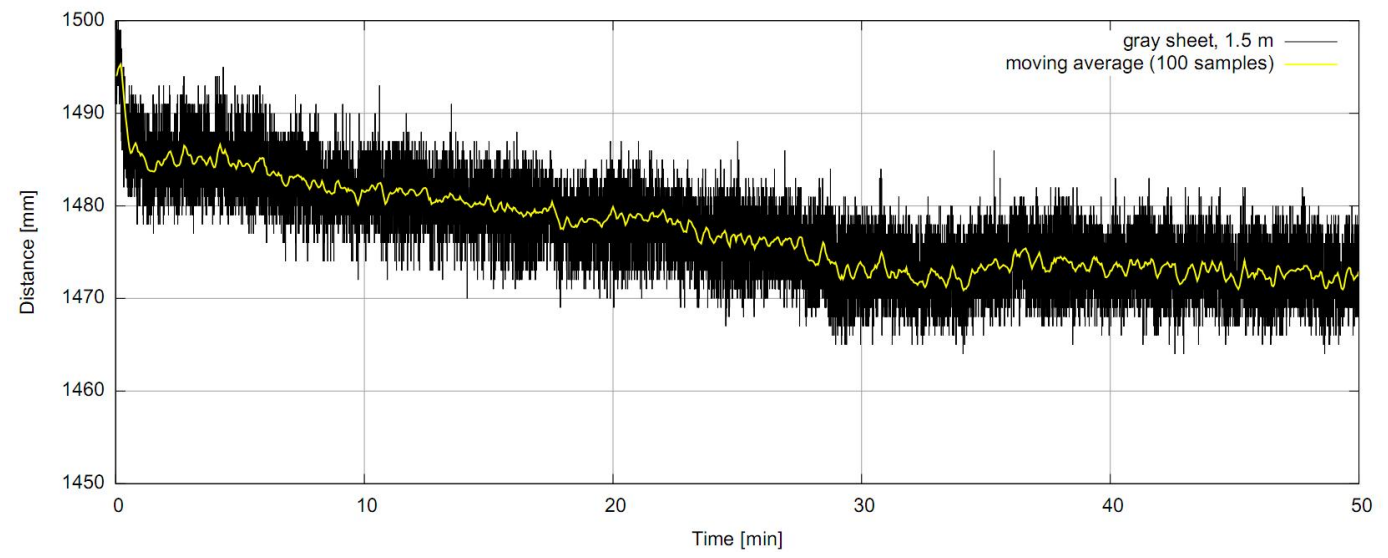

Fig. 3. Continuous drop of the measured distance due to heat dissipation of the sensor.

This definition may be different from the one used in the specification sheet of the sensor and hard to apply due to a missing knowledge about the null reference for the determination of the nominal distance. Nevertheless, it gives us a good impression of the sensor's measurement accuracy. In the following experiments, the center of the sensor indicated by the origin in Figure 2 defines the null-reference.

\section{Characteristics of The Hokuyo URG-04LX}

This section presents all the experiments and the resulting characteristics of the Hokuyo URG-04LX. The paper analyzes drift effects, influence of sensor orientation, ambient light effects and dependency on surface color, brightness and material. Finally, the section ends by checking the influence of target distance and incidence angle of the laser beam. For good comparison with characteristics of the Sick LMS 200, all measurements unrelated to distance influence are done at a fixed distance of $1.5 \mathrm{~m}$. Results for the Sick LMS 200 at $1.692 \mathrm{~m}$ are available from the paper of Ye and Borenstein [3]. If the experiment is not measuring the dependency on incidence angle, the latter is fixed to $0^{\circ}$. Other distances and incidence angles are always indicated. The standard target in sections IV-B, IV-C and IV-D is a gray sheet of paper, a surface that does not possess any distinctive properties regarding color or brightness. The standard measurement value is angle index 387 in the sensor's field of view, which is at $0^{\circ}$ and represents the distance straight ahead to the target surface. A similar experiment to Ye and Borenstein [3] was done to verify that the index that delivers the minimum distance is in fact 387 and not 384 , as indicated by the sensor's specification. Consequently, the angular error of the complete measurement setup including the error of the Hokuyo URG 04-LX corresponds to about $1^{\circ}$. In order to avoid this error, any user should do this test and reconfigure the "front-index" whenever the setup is changed.

\section{A. General effects}

The measurements showed that, without doing any changes to the experiment configuration, the considered index sometimes returns faulty measurements. During 5000 measurements, this occurs usually only once or twice, but the number of wrong measurements increases with a decreasing quality of the captured reflection of the laser beam, which obviously depends on the properties of the current surface as well as target distance and incidence angle of the beam. In the measurement software, outlier measurements are not taken into consideration but removed through a simple filter that disregards values outside of the possible measurement range of the Hokuyo. In addition, their number is counted. By this way, a bad influence of outlier values on the statistical evaluation could be avoided for proper characterization of the URG-04LX, still keeping an eye on the general functionality. The mixed pixels problem from [3] could not be verified. The measurement of a small object in front of a more distant background never lead to intermediate distance values, despite of sharp edges and several small position changes.

\section{B. Drift effects}

To get an impression of the importance of drift effects, a measurement of a gray sheet target surface at a distance of $1.5 \mathrm{~m}$ and under an incidence angle of $0^{\circ}$ has been performed for about 50 minutes (30'000 measurements). The result is shown in Figure 3. One recognizes a continuous drop of the measured distance of more than $10 \mathrm{~mm}$ over the first 30 minutes. The reason is probably a continuous increase of the operating temperature of the sensor due to the heat dissipation of the spindle motor. At the beginning of all the following measurements, the sensor was already operating for at least one hour, thus drift effects can be excluded from the corresponding results.

\section{Influence of the sensor's spatial orientation}

With the purpose to check if a rotation of the sensor has any significant influence on the measured value, measurements with a different orientation of the URG-04LX were performed. The orientation "laying right" means a rotation by $+90^{\circ}$, "laying left" a rotation by $-90^{\circ}$ and "upside-down" a rotation by $180^{\circ}$ (e.g. flipped) about the x-axis (defined in Fig. 2). Making these experiments with rotated Hokuyo 
sensor is quite simple since it only needs the complete level track to be rotated about its own axis.

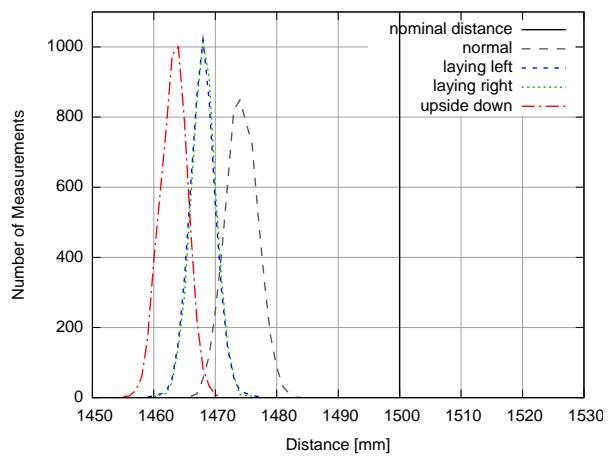

Fig. 4. Dependency on the orientation of the sensor.

Figure 4 shows that the measurement is clearly depending on the orientation of the sensor. The normal orientation results in a mean value of $1474 \mathrm{~mm}$. Both orientations "laying left" and "laying right" result in almost the same statistical distribution with a mean value of $1468 \mathrm{~mm}$. The similar behavior may be traced back to the fact that the effect in these two measurements is basically of the same nature and characterized by a perpendicular influence of the gravity force on the axis of rotation of the spindle motor. The upsidedown orientation of the Hokuyo results in an even worse deviation of the mean that drops down to $1464 \mathrm{~mm}$. The relative error increases from $-1.7 \%$ to $-2.4 \%$. This leads to the final recommendation to keep the sensor in the normal upstanding orientation whenever high precision is demanded. If the exact orientation is known and further characterization done with a more complex measurement setup that allows the exact configuration of the sensor orientation, a calibration model could be established helping to reduce the error when using the sensor for the realization of more demanding mobile robot applications.

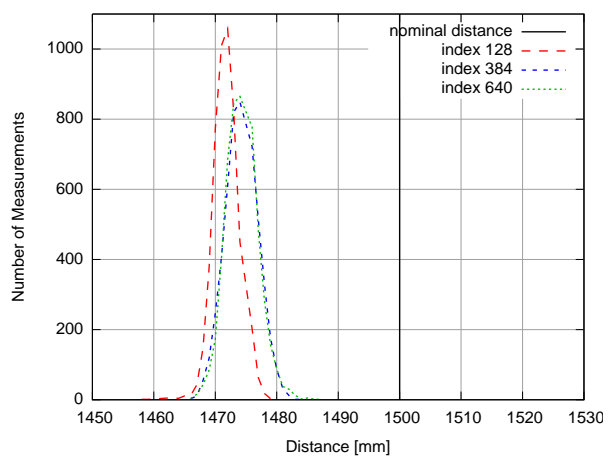

Fig. 5. Difference in measurement result if the same experiment is done by different index angles.

Another interesting point treats the question if there is a difference in the mean value if another angle index in the Hokuyo's field of view is considered for doing the measurement. For this, the Hokuyo's support on the level

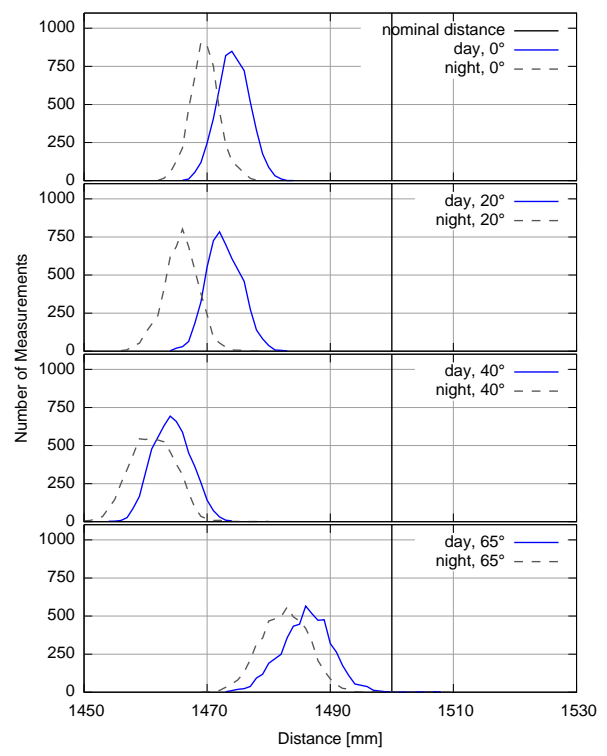

Fig. 6. Results of some measurements that have been executed in the dark for different incidence angles $\left(0^{\circ}, 20^{\circ}, 40^{\circ}, 65^{\circ}\right)$.

track has been designed to allow the sensor to be also mounted with a rotation of $\pm 90^{\circ}$ about the vertical axis. Figure 5 shows that for indices 384 and 640, the difference in the measurement stays negligible, and a slightly reduced mean value of $1472 \mathrm{~mm}$ for index 128 accompanied with a reduction of the standard deviation from $2.8 \mathrm{~mm}$ to $2 \mathrm{~mm}$. This behavior could be a sign for less reliability of the APD at the end of the scan due to a saturation of remaining charges of previously integrated photo current.

\section{Influence of missing ambient light}

Since the inspection robot related to the present work has to operate in dark regions, the effect of the absence of ambient light sources has to be checked. Therefore, some tests were repeated at night, a gray sheet measured at a distance of $1.5 \mathrm{~m}$ and with different incidence angles, and compared to the results obtained during the day. Figure 6 shows a comparison of the results. In particular, compared to the day measurements, it can be concluded that deviations in the mean values stay around $\pm 5 \mathrm{~mm}( \pm 0.33 \%)$ with no observable differences in standard deviation. A similar operation of the sensor in the dark can be expected.

\section{E. Effect of the surface color, surface brightness and mate- rial properties}

To get an idea of the influence of the surface color, brightness and material properties, the measurements of Ye and Borenstein [3] were repeated to have a base to compare the URG-04LX with the Sick LMS 200 and to complete the characterization in [2]. Three groups of experiments were defined: matted colors, gray levels and materials. The three base colors red, green and blue, 5 gray levels including black and white and in total 6 different materials for analyzing the 


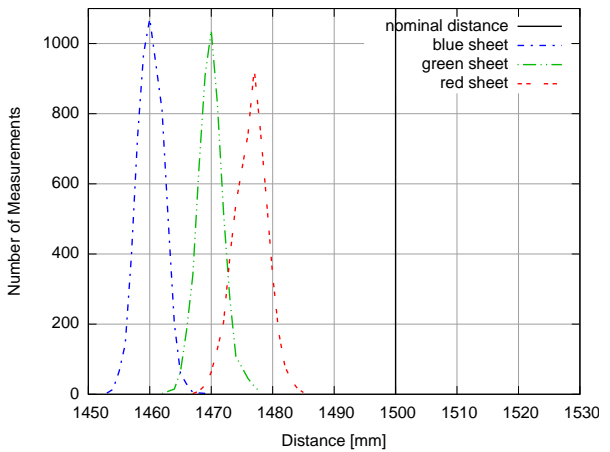

(a) surface color

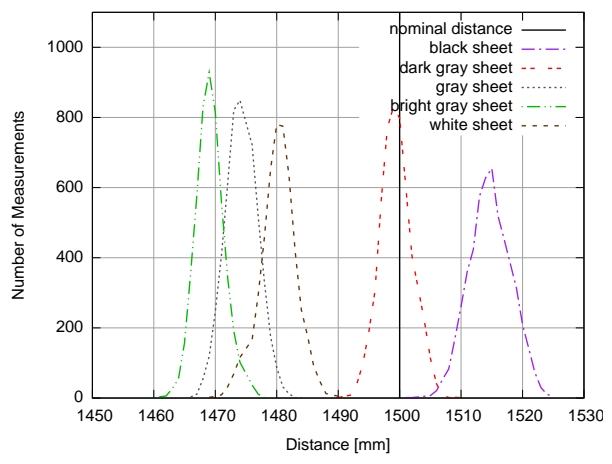

(b) surface brightness

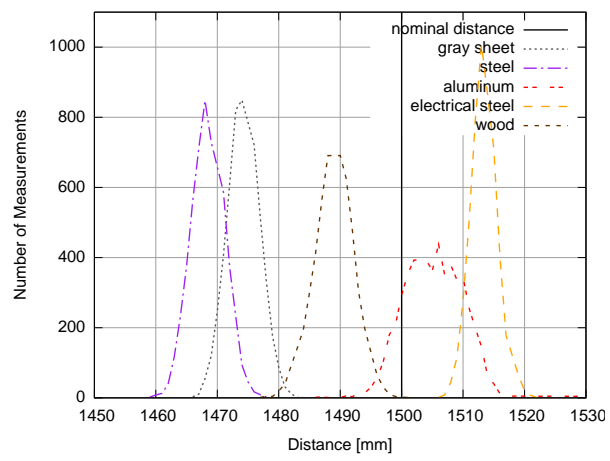

(c) material properties

Fig. 7. Effects of surface properties.

influence of the surface material resp. shininess were tested. The results are shown in Figure 7.

The mean values for red, green and blue are $1476 \mathrm{~mm}$, $1470 \mathrm{~mm}$ and $1460 \mathrm{~mm}$, the relative errors $-1.6 \%,-2 \%$ and $-2.7 \%$, and the standard deviations $2.8 \mathrm{~mm}, 2.1 \mathrm{~mm}$ and 2.3 mm respectively (cf. Figure 7(a)). A higher spread of the lobe goes along with an increase of the corresponding mean value.

This behavior cannot be observed on the basis of the measurement of the surface brightness effect (cf. Figure 7(b)). Although darker surfaces generally lead to higher mean values and elevated standard deviations of the resulting lobes, the brightest surface (e.g. white) is not leading to the lowest mean value or highest peak. The same is true for the case of the related sensor, the Sick LMS 200. Similar to the measurement of the color effect, deviations between the mean values are quite high and vary between $1469 \mathrm{~mm}$ for the bright gray target and $1514 \mathrm{~mm}$ for the black one $(-2.1 \%$ and $+1 \%)$. The corresponding standard deviations are relatively small between $2.3 \mathrm{~mm}$ and $3.4 \mathrm{~mm}$. Since deviations in the mean value are generally higher as with colored surfaces, the influence of the brightness of the surface must be considered as being more important than the color. As presented in the following section, a combination with elevated distances leads to very critical conditions. So far, there were no outlier values in the experiments.

The effect of the material properties and thus the surface roughness and shininess is hard to estimate regarding the huge influence of the surface brightness. As we will see in a later section, shiny surfaces in combination with a non-zero incidence angle quickly lead to missing functional compliance and faulty measurements. Since the incidence angle can never be exactly zero, this phenomenon makes it even harder to prove that shiny surfaces like aluminum lead to a more pronounced peak in the statistical distribution. We conclude that deviations in mean values are again quite high and free of any regularity (cf. Figure 7(c)). They vary between $1468 \mathrm{~mm}$ and $1513 \mathrm{~mm}$, which corresponds to relative errors of $-2.1 \%$ and $+0.9 \%$ respectively. The measured electrical steel is isolated with Carlite and therefore has a different surface texture than the normal steel.

\section{F. Dependency on target distance}

To measure the dependency on the target distance, 6 different surfaces were measured at distances varying between $91 \mathrm{~mm}$ and $4992 \mathrm{~mm}$. The results are presented in Figure 8. As the distance is growing, the diameter of the laser beam is obviously increasing and goes along with a continuous reduction of the intensity of the captured reflection. According to the behavior of the URG-04LX that we discovered in the previous sections, this leads in general to a growing difference between the measured and the nominal distance and an increment of the spreads. The results show a rather important dependency on the target distance. Exact relative errors will be presented in section V.

The phenomenon of multiple lobes observable in the results for the Sick LMS 200 appeared only for the smallest analyzed distance of $91 \mathrm{~mm}$. The nature of this behavior is hard to explain and may for example be the effect of parasitic reflections at the optical window of the sensor. Another effect that had to be asserted was the increasing number of wrong measurements with growing distance. A normal gray sheet is only measurable up to a distance of about $3.5 \mathrm{~m}$, whereas the distance to a white sheet can be measured up to the limits of the measurement range $(5.6 \mathrm{~m})$ (cf. Figure $8(\mathrm{~d}))$. A black sheet can no longer be captured at a distance above 2 $\mathrm{m}$. More shininess relieves this bad effect, but on the other hand requires a small incidence angle (cf. section IV-G). Consequently, the window for reliable measurements at high distances is very tiny for darker targets and the sensor should preferably be used for smaller distances and in combination with bright surfaces. 

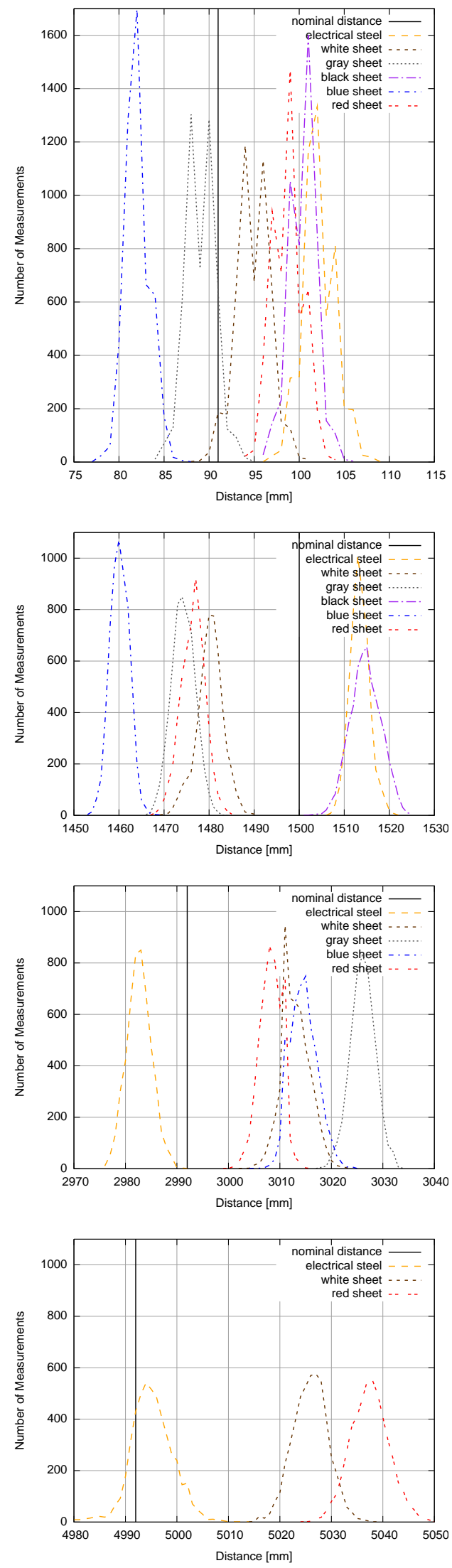

Fig. 8. Measurement results for different target distances (91, 1500, 2992 and $4992 \mathrm{~mm}$ ).

\section{G. Effect of the incidence angle}

As shown in Figure 9, the effect of the incidence angle is not comparable to previous results. If the incidence angle is different to zero, the main part of incident laser beam is obviously no longer reflected into the direction of the sensor. Thus standard deviations are increasing with growing incidence angle, but this time go along with decreasing mean values. Only beyond a certain limit value, the mean is again increasing. This limit value as well as the maximum incidence angles that still support a reliable measurement are clearly depending on the surface shininess. Whereas a gray sheet is captured up to incidence angles of $65^{\circ}$ and electrical steel up to $50^{\circ}$, a steel plate is no longer recognized after an incidence angle of only $10^{\circ}$. Aluminum does not support any incidence angle (100\% outliers). The overall impression leads to the conclusion that, supposing that the reflected laser beam is still strong enough, the effect of the incidence angle is less important than previously observed effects. As shown in the previous section, the reliability is also depending on the distance to the target, which clarifies that the effect of the incidence angle is even weaker if the same experiment is done but closer to the sensor. Exact relative errors can be found in the following section.

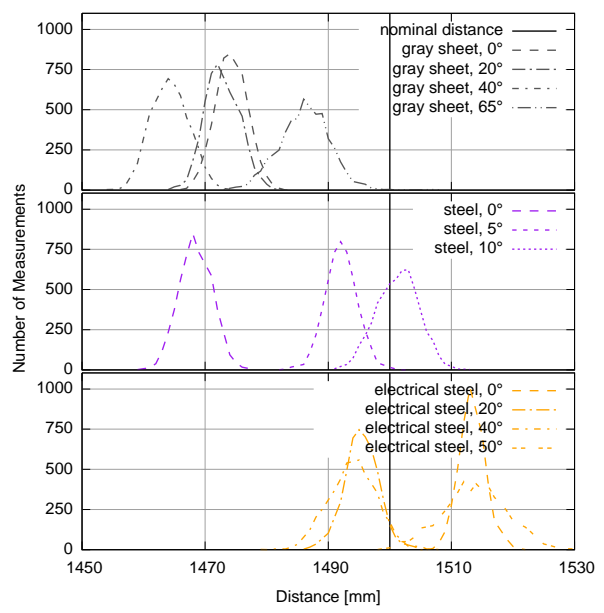

Fig. 9. Measurement results for different incidence angles at $1500 \mathrm{~mm}$.

\section{INVESTIGATION OF A CALIBRATION MODEL}

With respect to the work of Ye and Borenstein [3], this section tries to analyze the linearity and accuracy of the measurement results and establish a calibration model for the Hokuyo URG-04LX. It analyzes 6 distances and 7 incidence angles, each time averaging over 5000 measurement values. Given the rather small number of analyzed distances/angles, it is certainly true that this evaluation can not claim to provide an extended and very precise calibration model. However, this does not prevent it from giving a good idea of how the error behaves in function of these parameters. Figure 10 shows the absolute errors, which means the deviations between measured and nominal distance in function of distance or incidence angle. We can observe similarities in the 
overall curve progression of comparable surface materials, but a linear behavior is only true for small intervals of the error curves. Besides, we notice a huge dependency on the considered target surface properties. Compared to the Sick LMS 200, we have a much lower linearity in the absolute measurement errors. However, the major difference of all experiments is that the present work analyzes characteristics over the sensor's whole measurement range ( 0 to $5 \mathrm{~m}$ ). In the case of the Sick sensor, an investigation of target distances up to the limits of the measurement range ( $80 \mathrm{~m}$ in $\mathrm{cm}$ mode) could also result in reduced linearity.

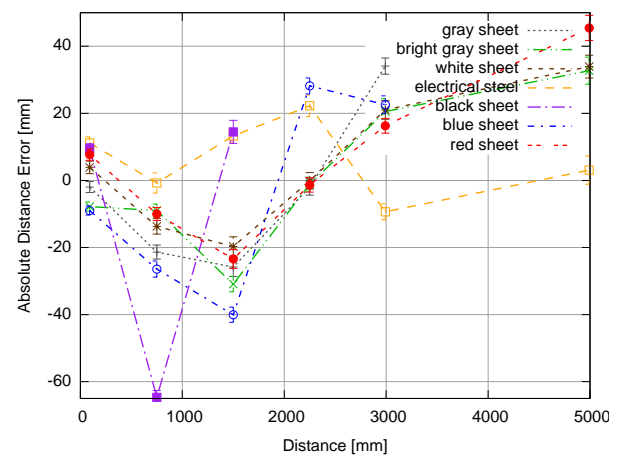

(a) Absolute distance error in function of distance.

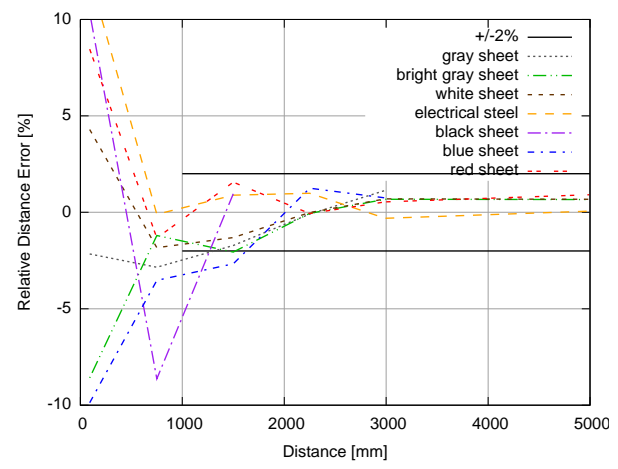

(b) Relative distance error in function of distance.

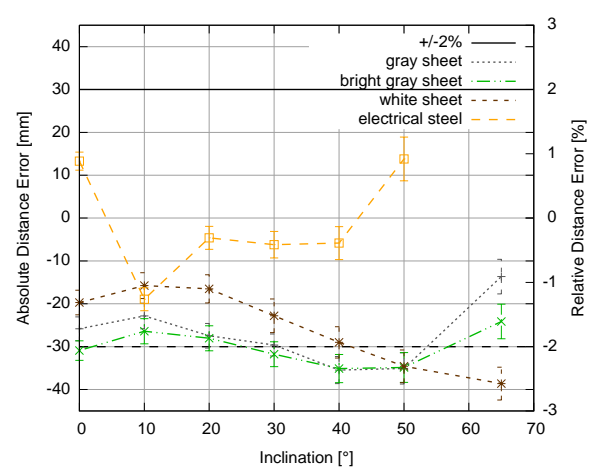

(c) Absolute and relative distance error in function of incidence angle (distance: $1500 \mathrm{~mm}$ ).

Fig. 10. Distance errors.

Concerning the measurement accuracy, Figure 10 shows that the relative errors stay within the region provided by the sensor's specification unless incidence angles are not getting too high. Remember that the specification is only valid for a white sheet target. A direct comparison with the accuracy indicated in the specification is complicated since not knowing the exact definition that the manufacturers used to compute the value. However, for the relative error in function distances above $1 \mathrm{~m}$, if not targeting a white sheet, one has to expect a relative error of about $2 \%$. It should also be emphasized that the investigated surfaces are not representing the most difficult cases for the sensor and relative errors may be higher if measuring for example a blue or a black sheet.

In practice, the application of a calibration model that respects the incidence angle is very complicated and frequently goes along with a reduced knowledge about the target surface properties. Therefore, the establishment of such a model is not very reasonable and hardly leads to an essential reduction of the absolute measurement error. We will go for a calibration model that relieves the error in function of the measured target distance. Only bright paper sheet targets are respected since values belonging to other materials or dark targets result in different curve progressions. Separated calibration models are better adapted for other target types. This increases the efficiency of the model for the considered targets. A simple polynomial regression of the third order according to the least squares method leads to the following expression for an estimation of the absolute distance measurement error $\Delta d=d_{\text {meas }}-d_{\text {nom }}$ :

$$
\begin{aligned}
\widehat{\Delta d}=\quad & -4.326 \cdot 10^{-9} \cdot d_{\text {meas }}^{3}+3.475 \cdot 10^{-5} \cdot d_{\text {meas }}^{2} \\
& -5.889 \cdot 10^{-2} \cdot d_{\text {meas }}+4.898
\end{aligned}
$$

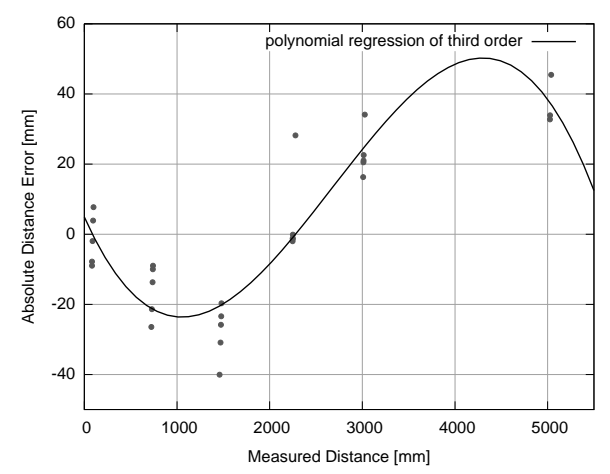

Fig. 11. Polynomial regression of third order of absolute distance error values.

This result with underlaying measurement values is shown in Figure 11. Resolving for an estimation of $d_{\text {nominal }}$, we obtain:

$$
\begin{aligned}
\widehat{d}= & 4.326 \cdot 10^{-9} \cdot d_{\text {meas }}^{3}-3.475 \cdot 10^{-5} \cdot d_{\text {meas }}^{2} \\
& +1.05889 \cdot d_{\text {meas }}-4.898
\end{aligned}
$$

Applying this calibration model to the previous measurement values results in smaller absolute errors, as shown in Figure 12. 


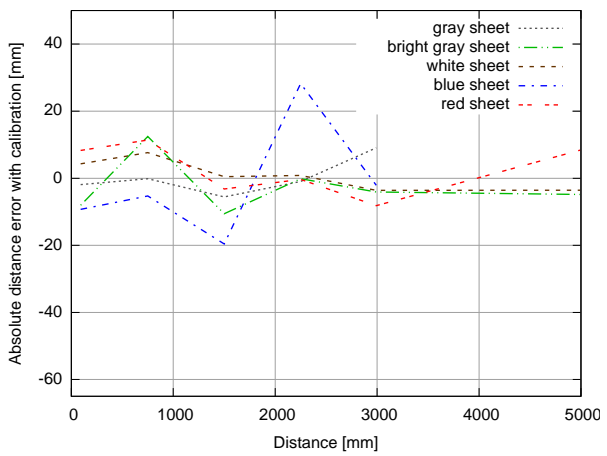

Fig. 12. Absolute distance error in function of distance with application of calibration model

\section{CONCLUSION}

This paper presents a characterization of the Hokuyo URG-04LX and analyzes various known issues of laser range finders like dependency on surface properties, distance of the target and incidence angle of the laser beam. The measurement results show that, in comparison to the Sick LMS 200, standard deviations are rather small and absolute deviations slightly higher (cf. Table I). Since both sensors have very different measurement ranges, it is hard to provide more direct juxtaposition. Instead, for further comparison, the reader is rather advised to directly consult the referred paper [3]. The URG 04-LX's accuracy is strongly depending on the surface brightness and material properties. Nonzero incidence angles may quickly lead to outlier values at elevated distances. For high reliability up to $5 \mathrm{~m}$, the sensor should preferably be used to measure bright and not too shiny targets. The accuracy provided by the specification of the sensor respects only the targeting of a white sheet of paper. Following the definition provided in this paper, the measurement accuracy (relative error) corresponds to $2 \%$. Some rather uncommon tests to check the influence of different angle indices as well as the effect of the absence of ambient light did not reveal significant errors. Only the measurement of the drift could manifest another weakness of the sensor and showed a continuous drop of the measurement value over the first 30 minutes of operation. Besides, the

\begin{tabular}{|l|c|c|c|c|}
\hline & \multicolumn{2}{|c|}{ URG-04LX } & \multicolumn{2}{c|}{ LMS 200 } \\
\hline sheet color & $\eta$ & $\sigma$ & $\eta$ & $\sigma$ \\
\hline white & -19.7 & 2.9 & 7.4 & 3.0 \\
bright gray & -30.9 & 2.3 & 6.7 & 4.1 \\
gray & -25.8 & 2.8 & 6.3 & 5.1 \\
dark gray & -0.8 & 2.6 & 5.6 & 3.5 \\
black & 14.5 & 3.4 & 10.7 & 3.5 \\
red & -23.4 & 2.8 & 9.4 & 1.6 \\
green & -30.2 & 2.1 & 10.3 & 3.2 \\
blue & -40.0 & 2.3 & 6.3 & 3.4 \\
\hline
\end{tabular}

TABLE I

COMPARISON OF ABSOLUTE ERRORS AND STANDARD DEVIATIONS [MM] BETWEen THE HOKUYO URG-04LX AND THE SICK LMS 200 AT A

DISTANCE OF ABOUT $1.5 \mathrm{M}$ returned value is strongly depending on the orientation of the sensor, but this is only a handicap if used in applications with rather special requirements similar to those of the mentioned tube inspection robot.

This paper also presents a calibration model for reducing the absolute measurement error of the distance values. However, the polynomial regression of the third order has been established using target surfaces with similar material properties (e.g. paper sheet). This means that, as already indicated by the measurement results for the electrical steel, the calibration model does not offer the same gain in accuracy for other materials or very dark targets.

Finally, we can conclude that, regarding the size, the Hokuyo URG-04LX surely stays a very interesting alternative to the Sick LMS 200 for localization and mapping tasks in mobile robot applications, even if the compact design suffers from loss of measurement accuracy. Regarding the latter, the URG-04LX shows a higher dependency on surface properties and measurement conditions than the Sick LMS 200, but stays competitive with respect to the precision. Especially for mapping tasks in compact robot applications, the URG-04LX becomes a very interesting solution.

\section{REFERENCES}

[1] F. TÂCHE, W. FISCHER, R. SIEGWART, R. MOSER, F. MONDANA, Compact Magnetic Wheeled Robot with High Mobility for Inspecting Complex Shaped Pipe Structures, Proceedings of the 2007 IEEE/RSJ International Conference on Intelligent Robots and Systems (IROS'07), p. 261-266, San Diego, USA, (2007)

[2] H. KAWATA, A. OHYA, S. YUTA, W. SANTOSH, T.MORI, Development of ultra-small lightweight optical range sensor system, Proceedings of the 2005 IEEE/RSJ International Conference on Intelligent Robots and Systems (IROS'05), p. 1078-1083, Edmonton, Canada, (2005)

[3] C. YE, J. BORENSTEIN, Characterization of a 2-D Laser Scanner for Mobile Robot Obstacle Negotiation, Proceedings of the 2002 IEEE International Conference on Robotics and Automation (ICRA apos '02), vol. 3, p. 2512-2518, Washington DC, USA, (2002)

[4] T. UEDA, H. KAWATA, T. TOMIZAWA, A. OHYA, S. YUTA, Mobile SOKUIKI Sensor System: Accurate Range Data Mapping System with Sensor Motion, The 2006 International Conference on Autonomous Robots and Agents, Palmerston North, New Zealand, (2006)

[5] W. C. STONE, M. JUbertS, N. DAGAlakis, J. STONE, J. GORMAN, Performance Analysis of Next-Generation LADAR for Manufacturing, Construction, and Mobility, National Institute of Standards and Technology, Gaitherburg, Maryland, (2004)

[6] P. STEINHAUS, R. DILLMANN, Aufbau und Modellierung des RoSi Scanners zur 3D-Tiefenbildakquisition, Autonome Mobile Systeme 2003 (AMS 2003): 18. Fachgesprch, Karlsruhe, Germany, (2003)

[7] H. SURMANN, K. LINGEMANN, A. NÜCHTER, A 3D Laser Range Finder for Autonomous Mobile Robots, Proceedings of the International Symposium on Robotics, Zurich, Switzerland, (2001)

[8] J. W. WEINGARTEN, G. GRUENER, R: SIEGWART, A Stateof-the-Art 3D Sensor for Robot Navigation, Proceedings of the 2004 IEEE/RSJ International Conference on Intelligent Robots and Systems (IROS'04), vol. 3, p. 2155-2160, Sendai, Japan, (2004)

[9] M. ALWAN, M. B. WAGNER, G. WASSON, P. SHETH, Characterization of Infrared Range-Finder PBS-03JN for 2-D Mapping, Proceedings of the 2005 IEEE International Conference on Robotics and Automation (ICRA'05), p. 3947-3952, Barcelona, Spain, (2005)

[10] K-H. LEE, R. EHSANI, Comparison of two $2 D$ laser scanners for sensing object distances, shapes, and surface patterns, Computers and Electronics in Agriculture 60 (ELSEVIER), p. 250-262, (2008)

[11] Hokuyo URG-04LX datasheet, Hokuyo Automatic Co., Ltd., Japan 